\title{
miR-122-5p suppresses the oncogenesis of PTC by inhibiting DUSP4 expression
}

\author{
NING HU ${ }^{1}$, YANHUA TIAN ${ }^{2}$, YANMEI SONG ${ }^{3}$ and LEILEI ZANG ${ }^{4}$ \\ ${ }^{1}$ Department IV of General Surgery and ${ }^{2}$ Department II of Oncology, The Second Hospital of Hebei Medical University, \\ Shijiazhuang, Hebei 050005; ${ }^{3}$ Department of Infection Management/Public Health, Hebei People's Hospital, \\ Shijiazhuang, Hebei 050057; ${ }^{4}$ Department V of General Surgery, The Second Hospital \\ of Hebei Medical University, Shijiazhuang, Hebei 050005, P.R. China
}

Received May 13, 2020; Accepted January 27, 2021

DOI: $10.3892 / \mathrm{mmr} .2021 .12007$

\begin{abstract}
MicroRNAs (miRNAs or miRs) play an important role in regulating the occurrence and development of papillary thyroid carcinoma (PTC). miR-122-5p is widely considered a tumour inhibitor, which has not been fully explored in PTC. Bioinformatics analysis identified dual specificity phosphatase 4 (DUSP4), a tumour promoter gene for PTC, as a downstream target of miR-122-5p. The aim of the present study was to investigate the role and molecular mechanism of miR-122-5p in PTC oncogenesis. In this study, the expression pattern of miR-122-5p in PTC cancer tissues and PTC cell lines was investigated via reverse transcription-quantitative PCR. Furthermore, the roles of miR-122-5p in PTC were explored using gain-of-function and loss-of-function assays. The results revealed that the expression of miR-122-5p was significantly lower in PTC cancer tissues, especially in cancer tissues with significant invasion or metastasis. Overexpression of miR-122-5p caused by miR-122-5p mimics inhibited the proliferation, invasion, and migration of the PTC cell line $\mathrm{K} 1$, while knockdown of miR-122-5p by miR-122-5p inhibitors exhibited the opposite effect. Furthermore, in vivo assays revealed that miR-122-5p overexpression inhibited tumour growth. In addition, miR-122-5p was negatively correlated with DUSP4 expression in PTC cancer tissues. miR-122-5p overexpression inhibited DUSP4 expression in $\mathrm{K} 1$ cells, while miR-122-5p downregulation produced the inverse effect.
\end{abstract}

Correspondence to: Professor Leilei Zang, Department V of General Surgery, The Second Hospital of Hebei Medical University, 215 Heping West Road, Xinhua, Shijiazhuang, Hebei 050005, P.R. China

E-mail: zllhbmu@sina.com

Abbreviations: PTC, papillary thyroid carcinoma; $\mathrm{CD}$, cluster of differentiation; IL, interleukin; FBS, fetal bovine serum; RT-qPCR, reverse transcription-quantitative PCR; CCK-8, Cell Counting Kit-8; IHC, immunohistochemistry

Key words: microRNA, microRNA-122-5p, dual specificity phosphatase 4, papillary thyroid carcinoma
Specifically, a luciferase reporter assay confirmed the binding sites of miR-122-5p on the 3'-UTR of DUSP4, demonstrating the targeting effect of miR-122-5p on DUSP4. miR-122-5p inhibited the oncogenesis of PTC by targeting DUSP4, revealing the potential application value of miR-122-5p in the diagnosis and treatment of PTC.

\section{Introduction}

The incidence of papillary thyroid carcinoma (PTC) is highest in patients with thyroid cancer, reaching $70-80 \%$ (1). The main clinical manifestation of PTC is a slowly growing thyroid mass with multifocality and a propensity for regional lymph node metastasis (2). Some PTCs are highly aggressive; some tend to dedifferentiate and eventually develop into poorly differentiated or undifferentiated carcinomas, leading to a decreased survival rate and compromised quality of life (2). Therefore, it is important to further improve the cure rate and survival rate of patients with PTC to study the growth, invasion and metastasis of PTC cells and explore the molecular mechanism, on the basis of which it would be possible to find biomarkers with differential expression and metastatic prediction in PTC, as well as molecules that contribute to therapy. An increasing number of studies have revealed that aberrant expression of microRNAs (miRNAs) plays an important role in regulating the growth and metastasis of PTC $(3,4)$. Therefore, by studying the biological function and mechanism of miRNAs in PTC, new insights can be provided for the diagnosis and treatment of PTC.

It is widely accepted that miR-122-5p has a tumour suppressive function in a variety of carcinomas such as hepatocellular carcinoma, bile duct carcinoma and gastric cancer (5-7). However, the role of miR-122-5p in PTC has not been clarified. Dual specificity phosphatase 4 (DUSP4) is a member of the dual specificity phosphatase family and could negatively regulate the activity of the MAP kinases (MAPK) (8). DUSP4 has been widely recognized as a biological marker of multiple malignant tumours. Alterations in DUSP4 expression are involved in the oncogenesis of a variety of tumours. DUSP4 acts as a tumour suppressor in most cancers (8-10) but contributes to the occurrence and development of a small portion of tumours, including PTC $(7,11,12)$. A previous study revealed that DUSP4 expression in PTC was significantly higher 
than that in adjacent normal tissues (12). Moreover, the high expression of DUSP4 was not only related to lymph node metastasis and extrathyroidal extension of PTC but also an independent risk factor for lymph node metastasis. In addition, DUSP4 expression was associated with BRAF mutations in PTC cancer tissues and cell lines (12). Therefore, DUSP4 is considered a potential biomarker for the pathogenesis of PTC. Through a bioinformatics study, it was revealed that the 3'-UTR of DUSP4 mRNA has a specific binding sequence for miR-122-5p (7). Xu et al (7) revealed for the first time that miR-122-5p inhibited the migration, invasion and metastasis of gastric cancer (GC) cells by downregulating DUSP4. In summary, it is hypothesized that miR-122-5p inhibits tumour development by inhibiting DUSP4 in PTC cells.

The present study aimed to explore the role of miR-122-5p in PTC oncogenesis. The expression pattern of miR-122-5p in PTC cancer tissues and PTC cell lines was investigated, and the roles of miR-122-5p in PTC were explored.

\section{Materials and methods}

Clinical tissue specimens. A total of 45 pairs of PTC tissues and adjacent non-cancerous tissues were obtained with written informed consent via surgical resection at the Second Hospital of Hebei Medical University (Shujiazhuang, China) between January 2016 and February 2019. The age range of the patients was 25-77 years, and the median age was 52 years. There were 16 male patients and 29 female patients. The distance range between the tumour samples and adjacent non-cancerous tissues was between 1.3 and $2.3 \mathrm{~cm}$. All tissue specimens were histopathologically examined by three independent pathologists. Thus, all subjects were confirmed as PTC. No chemotherapy or radiotherapy was performed for these patients before the surgery. Fresh tissue specimens were frozen in liquid nitrogen and stored at $-80^{\circ} \mathrm{C}$ until use. All the clinical samples were acquired with written informed consent from the participants. The Institutional Review Board of the Second Hospital of Hebei Medical University reviewed and approved the present study (approval no. 2016-R269). The clinical studies were conducted according to the principles expressed in the Declaration of Helsinki.

Cell lines and culture. The human normal thyroid epithelial cell line FRTL-5, thyroid cancer cell line BCPAP and PTC cell lines TPC-1, and K1 were obtained from the American Type Culture Collection. FRTL-5 was incubated in Keratinocyte Serum Free Medium (KSFM; Thermo Fisher Scientific, Inc.). TPC-1, K1 and BCPAP were incubated in RPMI-1640 medium (Thermo Fisher Scientific, Inc.) along with $10 \%$ fetal bovine serum (FBS; Thermo Fisher Scientific, Inc.). The whole cells were maintained in humidified atmosphere at $37^{\circ} \mathrm{C}$ with $5 \% \mathrm{CO}_{2}$. BCPAP and $\mathrm{K} 1$ were authenticated by STR profiling.

Reverse transcription-quantitative PCR (RT-qPCR) assays. The total RNA from PTC tissues, adjacent non-cancerous tissues and all cell lines was extracted and purified using TRIzol reagent (Thermo Fisher Scientific, Inc.). Synthesis of cDNA and RT-qPCR measurements were carried out as previously described $(13,14)$. The designed primer sequences for RT-qPCR were as follows: miR-122-5p,
5'-TATTCGCACTGGATACGACACAAAC-3' (sense) and 5'-GCCCGTGGAGTGTGACAATGGT-3' (anti-sense); DUSP4, 5'-CTACATCCTAGGTTCGGTCAAC-3' (sense) and 5'-TAGACGATGACCGCCGAGTA-3' (anti-sense); GAPDH, 5'-CCTGCCTCTACTGGCGCTGC-3' (sense) and 5'-GCA GTGGGGACACGGAAGGC-3' (anti-sense). Relative expression was calculated using the $2^{-\Delta \Delta \mathrm{Cq}}$ method (15).

GAPDH was used as the internal reference. RT-qPCR was performed using SYBR Premix Ex Taq $^{\mathrm{TM}}$ kit (Takara Bio, Inc.) and ABI7500 PCR system (Applied Biosystems; Thermo Fisher Scientific, Inc.).

Plasmid construction and transfection. miR-122-5p full-length sequences were PCR amplified using Phusion HSII Flash High-Fidelity PCR Master Mix (Thermo Fisher Scientific, Inc.) according to the manufacturer's protocol. miR-122-5p mimic (5'-UGGAGUGUG ACA AUGGUGUUUG-3'), miR-mimic control (5'-GUGCACGAAGGCUCAUCAUU-3'), miR-122-5p inhibitor (5'-AACACCAUUGUCACACUCCAU U-3') and miR-inhibitor control (5'-UUCUCCGAACGUGUC ACGUTT-3') were obtained from Shanghai GenePharma Co., Ltd. miRNA mimics, miRNA inhibitors, miRNA mimics control and miRNA-inhibitors control in serum-free Accell ${ }^{\mathrm{TM}}$ medium (Waters Corporation) were transfected into K1 cells at a final concentration of $50 \mathrm{nM}$ using Lipofectamine ${ }^{\circledR}$ 2000 (Invitrogen; Thermo Fisher Scientific, Inc.) according to the manufacturer's protocols. Incubation was performed for $24 \mathrm{~h}$ at $37^{\circ} \mathrm{C}$, and then transfected cells were transferred to RPMI-1640 medium. All tests were performed $72 \mathrm{~h}$ after transfection. The transfection efficiency of plasmids was identified by qPCR.

Assessment of cell viability. To assess cell proliferation, Cell Counting Kit-8 (CCK-8) assays were performed. For CCK-8 assays, the $\mathrm{K} 1$ cells in each group were plated into 96-well plates with 2,000 cells/well. At the indicated time-points, $10 \mu$ l CCK-8 reagent (Dojindo Molecular Technologies, Inc.) was added into the treated cells. Following incubation for $2 \mathrm{~h}$ at $37^{\circ} \mathrm{C}$, the optical density at $450 \mathrm{~nm}(\mathrm{OD} 450)$ was measured using Varioskan Flash reader (Thermo Fisher Scientific, Inc.).

Cell invasion and migration assays. Cell invasion was assessed using a Transwell assay and cell migration was measured using a scratch/wound healing assay. For the Transwell assay, cells $\left(1 \times 10^{5}\right.$ cells/well) suspended in serum-free DMEM supplemented with $1 \mathrm{mg} / \mathrm{ml}$ mitomycin C (inhibitor of cell proliferation) were seeded onto the upper chamber of the Transwell inserts (24-well insert, pore size $8 \mathrm{~mm}$ ). The filter membranes were pre-coated with Matrigel at $37^{\circ} \mathrm{C}$ for $30 \mathrm{~min}$. DMEM containing 20\% FBS was added to lower chamber. After $36 \mathrm{~h}$ of incubation, the cells migrating into lower surface of the inserts were fixed for $30 \mathrm{~min}$, stained with $1 \%$ crystal violet at $37^{\circ} \mathrm{C}$ for $30 \mathrm{~min}$, and photographed under an inverted light microscope (Olympus Corporation; scale bar, $100 \mu \mathrm{m}$ ). Invasion was measured by counting the number of stained cells.

For the scratch/wound healing assay, cells ( $1 \times 10^{5}$ cells/well) were seeded in 12-well plates and cultured until $75 \%$ confluence. Then, a wound was created by manually scratching the cell monolayer with a $200-\mu 1$ pipette tip. The cultures were washed to remove floating cells, and then the adherent cells 

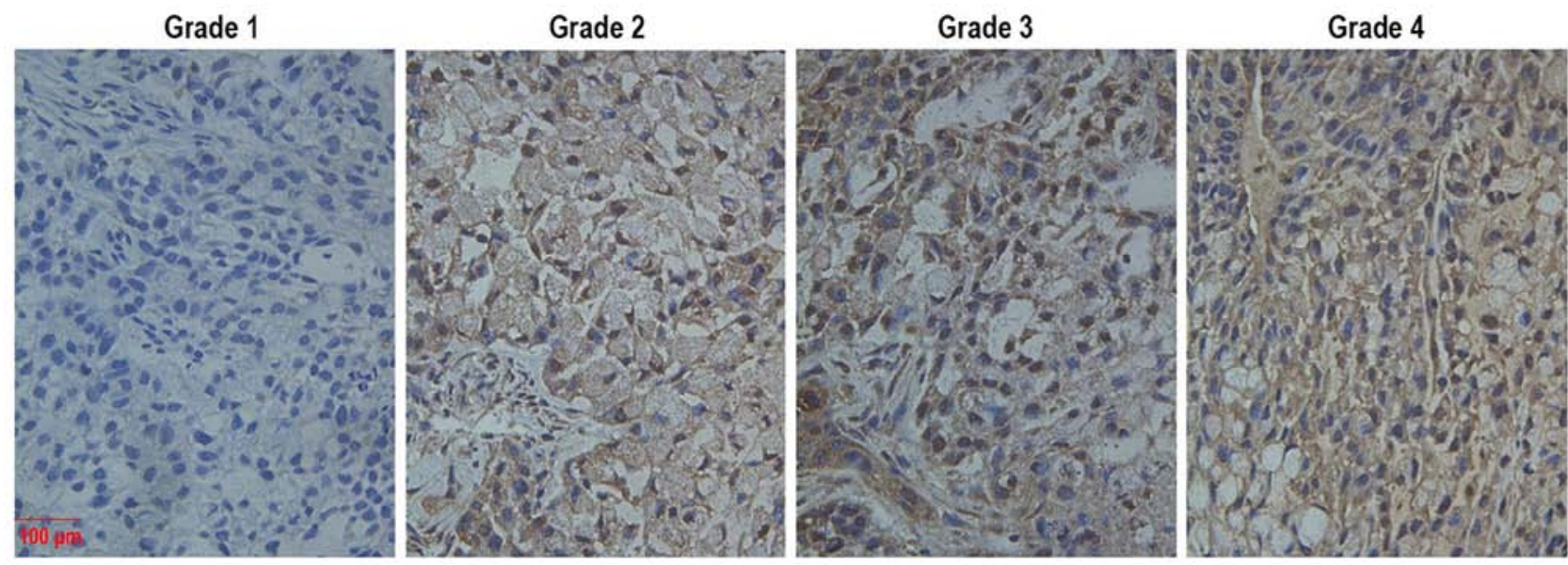

Figure 1. Immunohistochemical images with different dual specificity phosphatase 4 staining intensities in patients with papillary thyroid carcinoma. Scale bar, $100 \mu \mathrm{m}$.

were incubated in serum-free DMEM. Cell migration into the wound was observed under an inverted light microscope (Olympus Corporation; scale bar, $200 \mu \mathrm{m}$ ) at 0 and $24 \mathrm{~h}$ for each group. The scratch area was calculated using ImageJ software (v1.8.0; National Institutes of Health).

Western blotting. Whole-cell lysate protein from cells with indicated interventions in 6-well plates were extracted using ice-cold RIPA lysis buffer (Beyotime Institute of Biotechnology). The protein content was assessed using the BCA method. The concentration range of protein loaded per well was 5.2-6.7 $\mu \mathrm{g} / \mu \mathrm{l}$. A total of $40 \mu \mathrm{g}$ protein of each sample was separated via SDS-PAGE on $10 \%$ gels, and subsequently separated proteins were transferred onto polyvinylidene fluoride (PVDF) membranes. After being blocked with 5\% non-fat milk for $1 \mathrm{~h}$ at room temperature, PVDF membranes were incubated with the antibodies against DUSP4 (1:10,000; cat. no. 5149) and $\beta$-tubulin (1:10,000; cat. no. 2146) (both from Cell Signaling Technology, Inc.) overnight at $4^{\circ} \mathrm{C}$. Then, a HRP-conjugated secondary antibody $(1: 10,000$; cat. no. SA00001-1; Wuhan Sanying Biotechnology) was used at room temperature for $1 \mathrm{~h}$. The bands were visualized using enhanced chemiluminescent substrate reagent kit (Amersham; Cytiva) and chemiluminescence system (Amersham Image 600; General Electric; Cytiva). The signal densitometry was semi-quantified using ImageJ software (v1.8.0; National Institutes of Health).

Immunohistochemistry (IHC). The tumor tissues were fixed in $4 \%$ paraformaldehyde buffered with phosphate-buffered saline for $24 \mathrm{~h}$ at room temperature, embedded in paraffin and serially sectioned $(4 \mu \mathrm{m})$. Next, the tissue sections were blocked in 5\% goat serum (Wuhan Servicebio Technology Co., Ltd.) for $15 \mathrm{~min}$ at room temperature, and incubated with primary DUSP4 antibody (1:100; cat. no. ab72593; Abcam) at $4^{\circ} \mathrm{C}$ overnight. The sections were incubated with HRP-conjugated anti-rabbit (1:200; cat. no. GB23303; Wuhan Servicebio Technology Co., Ltd.) at $37^{\circ} \mathrm{C}$ for $20 \mathrm{~min}$, and then visualized using a PV-9000 DAB detection kit according to the manufacturer's protocol. The sections were counterstained with hematoxylin for $3 \mathrm{~min}$ at room temperature and observed under a light microscope (Olympus Corporation; scale bar, $100 \mu \mathrm{m})$. DUSP4 staining was graded semi-quantitatively as previously described (16). Staining intensity was graded as 1 (no staining), 2 (weak staining), 3 (clear staining), or 4 (strong staining). The IHC images regarding Grade 1-4 tumour tissues are presented in Fig. 1. The intensity and abundance (expressed as a fraction) were multiplied to obtain the total immunoreactivity score.

Animal experiments. A total of 30 male athymic BALB/c nude mice (6 weeks old; 19-22 g) were obtained from the Shanghai SLAC Laboratory Animal Co., Ltd. miR-122-5p mimic-transfected and control K1 cells were inoculated subcutaneously on the ventral side of the right rib at the density of $2 \times 10^{6}$ cells per mouse (10 mice per group). All mice were divided into two groups: Control group and the miR-122-5p mimics group. After 30 days, tumour-bearing mice were anesthetized with ether ( $6 \mathrm{ml}$; inhalation anesthesia), and then sacrificed via cervical dislocation. The sacrifice of mice was confirmed when the heart and breathing stopped. Subsequently, all tumours were removed and weighed. Tumour volumes were calculated using the following formula: Volume $\left(\mathrm{cm}^{3}\right)=$ length $(\mathrm{L} ; \mathrm{cm}) \mathrm{x}$ width $(\mathrm{W} ; \mathrm{cm})^{2} / 2$. The largest tumour volume was $\sim 1.5 \mathrm{~cm}^{3}$. Athymic nude mice assays were conducted in accordance with the Institutional principles for the concern and use of animals, and the corresponding protocols (including euthanasia protocol) were approved by the Institutional Animal Care and Use Committee of the Second hospital of Hebei Medical University. The mice were housed in a common environment in which the room temperature was $\sim 20-30^{\circ} \mathrm{C}$ and the humidity $\sim 60-80 \%$ and fed a general laboratory diet.

Luciferase reporter assays. TargetScan (http://www. targetscan.org) was used to predict the presence of complementary binding sites between miR-122-5p and the sequence 3'-UTR of DUSP4. To establish the mutated DUSP4 reporter vector, the site-specific mutagenesis system (Thermo Fisher Scientific, Inc.) was used to mutate the complementary binding site. The wild-type (WT) 3'-UTR luciferase reporter plasmid (pMIR-DUSP4-WT) and mutant (MUT) reporter plasmid (pMIR-DUSP4-MUT) were subsequently constructed. The 

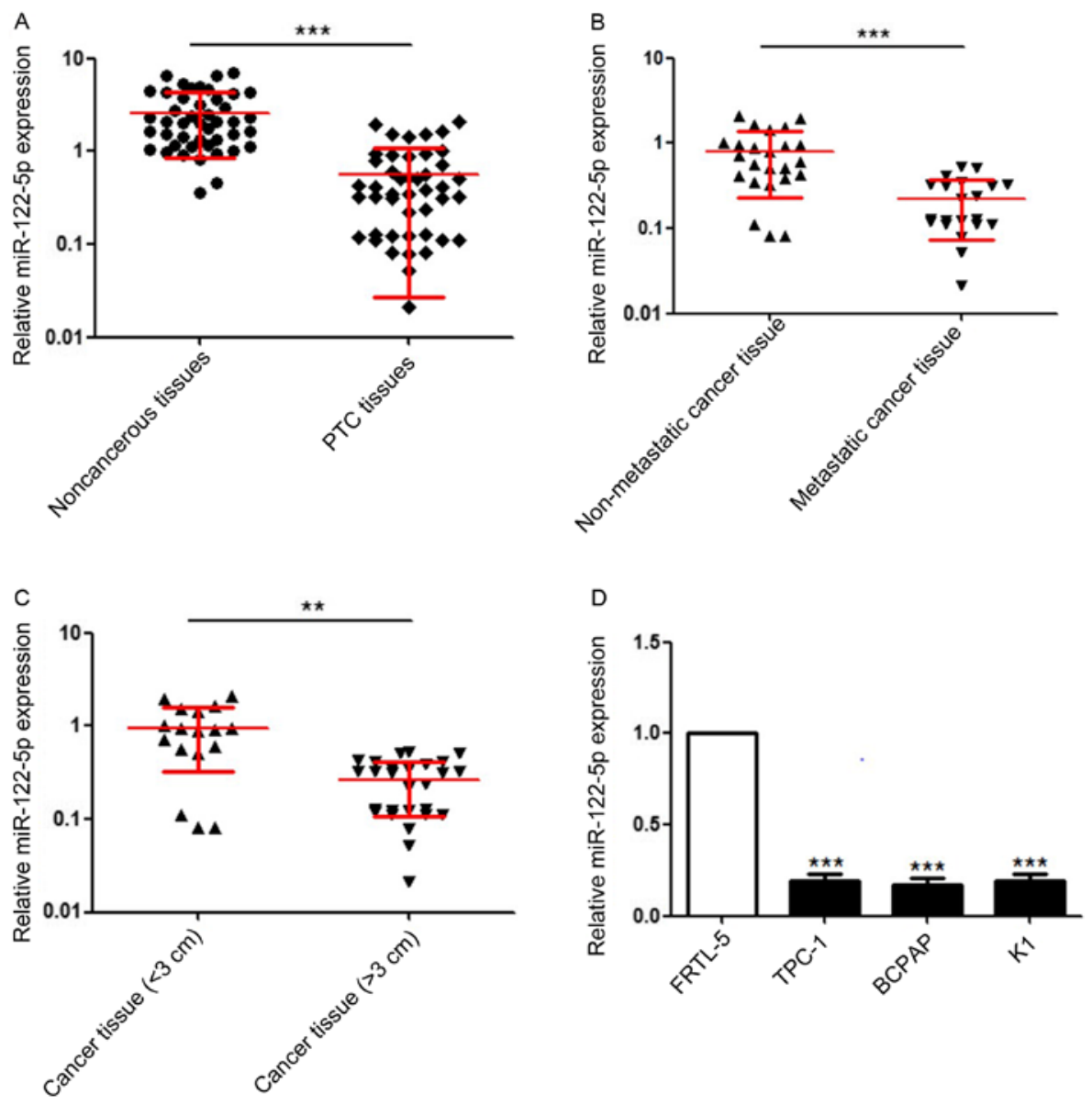

Figure 2. miR-122-5p is downregulated in PTC specimens. (A) miR-122-5p expression in 45 pairs of PTC tissues and adjacent non-cancerous tissues. ${ }^{* * *} \mathrm{P}<0.001$ by Wilcoxon signed-rank test. (B) miR-122-5p expression in 21 PTC specimens with metastasis and 24 non-metastatic PTC specimens. ${ }^{* * *} \mathrm{P}<0.001$ by Wilcoxon rank sum test. (C) miR-122-5p expression in 27 PTC specimens with diameters $>3 \mathrm{~cm}$ and 18 PTC specimens with diameters $<3 \mathrm{~cm}$. Results are presented as the median with interquartile range. ${ }^{* *} \mathrm{P}<0.01$ by Wilcoxon rank sum test. (D) miR-122-5p expression in normal thyroid epithelial cell line FRTL-5, thyroid cancer cell line BCPAP and PTC cell lines TPC-1, K1. Results are presented as the mean \pm SEM from three independent experiments. ${ }^{* * *} \mathrm{P}<0.001$ by one-way ANOVA. miR, microRNA; PTC, papillary thyroid carcinoma.

3'-UTRs (including WT and MUT) of DUSP4 was chemically synthesized and cloned into pMIR expression vectors including luciferase genes (pmiR-GLO Dual-Luciferase reporter vector; Promega Corporation) to construct pMIR-DUSP4-WT or pMIR-DUSP4-MUT plasmid as previously described (7). The sequences of miR-122-5p mimic and miR-mimic control were described above (Shanghai GenePharma Co.,Ltd.). miR-122-5p mimic or miR-mimic control, and pMIR-DUSP4-WT or pMIR-DUSP4-MUT were co-transfected into K1 cells using Lipofectamine $^{\circledast} 3000$ (Thermo Fisher Scientific, Inc.) for $48 \mathrm{~h}$. After transfection, luciferase activity in cell lysates was measured using a Dual-luciferase reporter system (Promega Corporation) according to the manufacturer's protocol. Relative luciferase intensity was normalized to Renilla luciferase activity.

Statistical analysis. All data are presented as the mean \pm SEM. The experiments were repeated at least three times. All statistical analyses were performed using the GraphPad Prism Software 6 (GraphPad Software, Inc.). For comparisons, Wilcoxon signed-rank test, Wilcoxon rank sum test, Pearson, one-way ANOVA or paired Student's t-test were performed as indicated. Pearson's correlation analysis was performed for correlation analysis. Pearson's chi-square test was performed to determine the association between miR-122-5p expression and clinicopathological characteristics. Bonferroni post hoc test was performed following one-way ANOVA. $\mathrm{P}<0.05$ was considered to indicate a statistically significant difference.

\section{Results}

miR-122-5p is downregulated in PTC specimens. miR-122-5p expression levels in 45 pairs of PTC tissues and adjacent non-cancerous tissues were detected by RT-qPCR. As revealed in Fig. 2A, miR-122-5p was significantly downregulated in PTC tissue specimens compared with adjacent non-cancerous tissues. The Pearson's chi-square test revealed that high miR-122-5p expression was negatively associated with higher CDFI classification (17), stronger invasion, larger PTC tumours and advanced pathological T/N stage (Table I). Moreover, 21 PTC specimens with metastasis had lower miR-122-5p expression than 24 non-metastatic PTC specimens (Fig. 2B). Accordingly, compared with 18 PTC specimens with diameters $<3 \mathrm{~cm}, 27$ PTC specimens with diameters $>3 \mathrm{~cm}$ exhibited lower expression of miR-122-5p (Fig. 2C). In addition, miR-122-5p expression levels in the normal thyroid 
Table I. Association between miR-122-5p expression levels and clinicopathological characteristics of patients with papillary thyroid carcinoma.

\begin{tabular}{|c|c|c|c|c|}
\hline \multirow[b]{2}{*}{ Characteristics } & \multirow[b]{2}{*}{ Cases } & \multicolumn{2}{|c|}{ miR-122-5p expression } & \multirow[b]{2}{*}{ P-value } \\
\hline & & Low & High & \\
\hline Total & 45 & 23 & 22 & \\
\hline Sex & & & & 0.5589 \\
\hline Male & 12 & 7 & 5 & \\
\hline Female & 33 & 16 & 17 & \\
\hline Age, years & & & & 0.3666 \\
\hline$\geq 65$ & 20 & 13 & 7 & \\
\hline$<65$ & 25 & 12 & 13 & \\
\hline CDFI classification grade & & & & $0.0080^{\mathrm{a}}$ \\
\hline $\mathrm{I}$ & 28 & 10 & 18 & \\
\hline II-III & 17 & 13 & 4 & \\
\hline Degree of invasion & & & & $0.0018^{\mathrm{a}}$ \\
\hline Intrathyroid & 22 & 6 & 16 & \\
\hline Extrathyroid & 23 & 17 & 6 & \\
\hline PTC diameter, cm & & & & $0.0001^{\mathrm{a}}$ \\
\hline$\geq 3.0$ & 27 & 21 & 6 & \\
\hline$<3.0$ & 18 & 2 & 16 & \\
\hline Pathological stage (T) & & & & $0.0027^{\mathrm{a}}$ \\
\hline $\mathrm{T} 2$ & 24 & 7 & 17 & \\
\hline T3-T4 & 21 & 16 & 5 & \\
\hline Pathological stage (N) & & & & $0.0305^{\mathrm{a}}$ \\
\hline No & 32 & 12 & 20 & \\
\hline N1 & 13 & 11 & 2 & \\
\hline Outcome & & & & 0.2150 \\
\hline Persistent/recurrent & 2 & 2 & 0 & \\
\hline Death & 1 & 1 & 0 & \\
\hline Cured & 42 & 20 & 22 & \\
\hline
\end{tabular}

miR-122-5p median expression level was used as the cut-off. P-values were acquired using Pearson's chi-square test. ${ }^{\text {a }}<00.05$ miR, microRNA; PTC, papillary thyroid carcinoma.

epithelial cell line FRTL-5, thyroid cancer cell line BCPAP and PTC cell lines TPC-1, K1 were detected by RT-qPCR. As presented in Fig. 2D, miR-122-5p was also significantly downregulated in PTC cell lines.

miR-122-5p inhibits the proliferation, invasion and migration of PTC. To explore the roles of miR-122-5p in PTC, miR-122-5p was overexpressed or silenced by transducing miR-122-5p mimics or miR-122-5p inhibitors into K1 cells. The transfection efficiency of plasmids was determined through the detection of mRNA levels (Fig. 3A). CCK-8 assays revealed that miR-122-5p overexpression reduced the proliferation of $\mathrm{K} 1$ cells (Fig. 3B). In addition, Transwell assays revealed that miR-122-5p overexpression inhibited cell invasion, and scratch assays revealed that miR-122-5p overexpression decreased the number of migratory cells (Fig. 3C-F). By contrast, miR-122-5p knockdown generated the opposite results in all the aforementioned parameters (Fig. 3B-F).
To explore the significance in vivo of miR-122-5p in PTC, miR-122-5p-overexpressing and control K1 cells were injected subcutaneously into nude mice. The weight of the xenograft tumours was measured 30 days after injection. As revealed in Fig. 3G and H, miR-122-5p overexpression significantly reduced the weights of PTC xenograft tumours.

miR-122-5p suppresses DUSP4 expression. Next, it was investigated whether the negative regulation of miR-122-5p on DUSP4 exists in vivo and in vitro. DUSP4 expression levels in the same 45 pairs of PTC tissues and adjacent non-cancerous tissues used in Fig. 2A were observed using IHC. As revealed in Fig. 4A and B, DUSP4 protein levels were significantly upregulated in PTC tissue specimens compared with adjacent non-cancerous tissues. The correlation analyses between DUSP4 expression and miR-122-5p expression in PTC tissues revealed that DUSP4 expression levels were negatively correlated with that of miR-122-5p in 

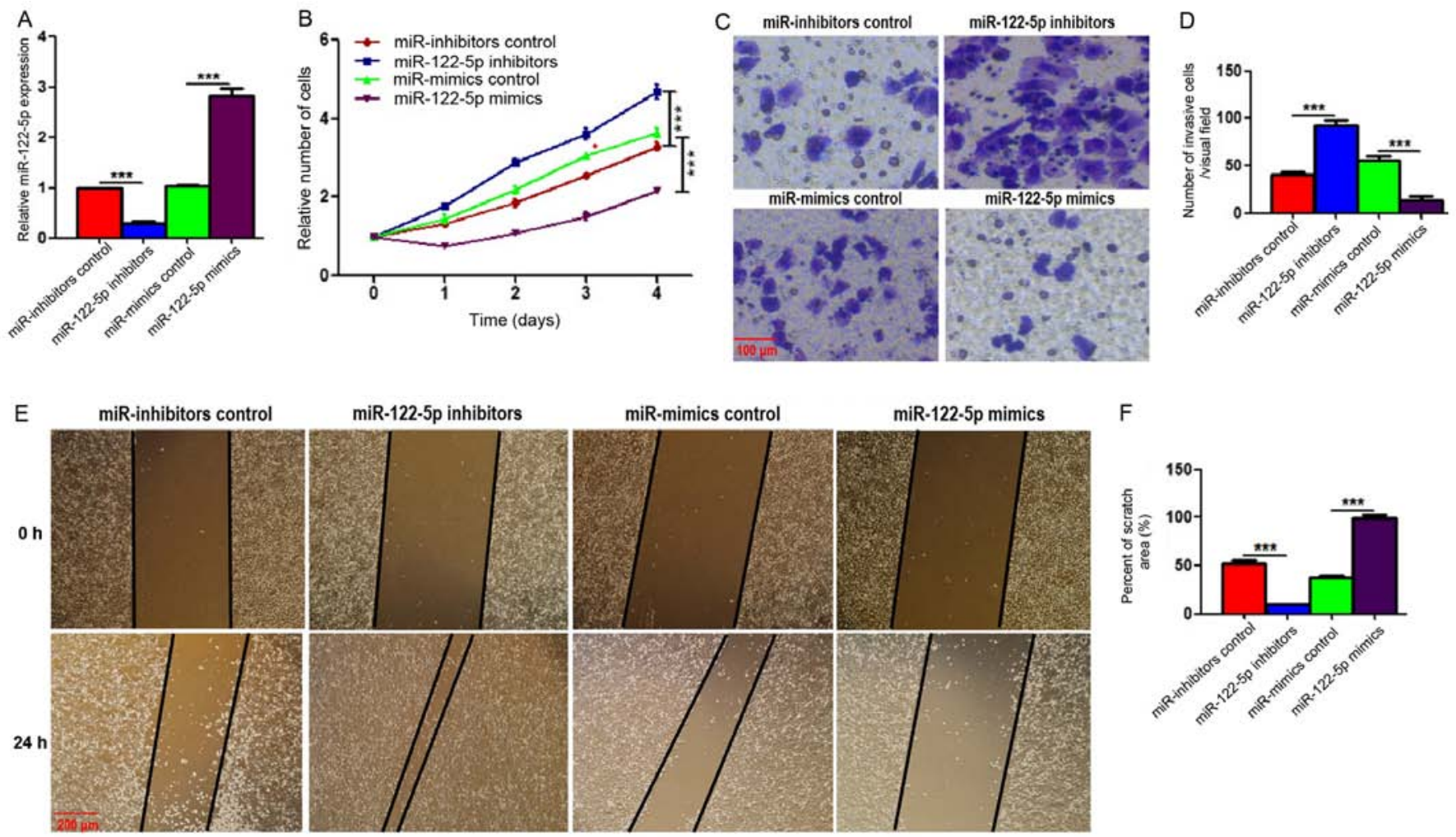

miR-122-5p mimics

$\mathrm{F}$
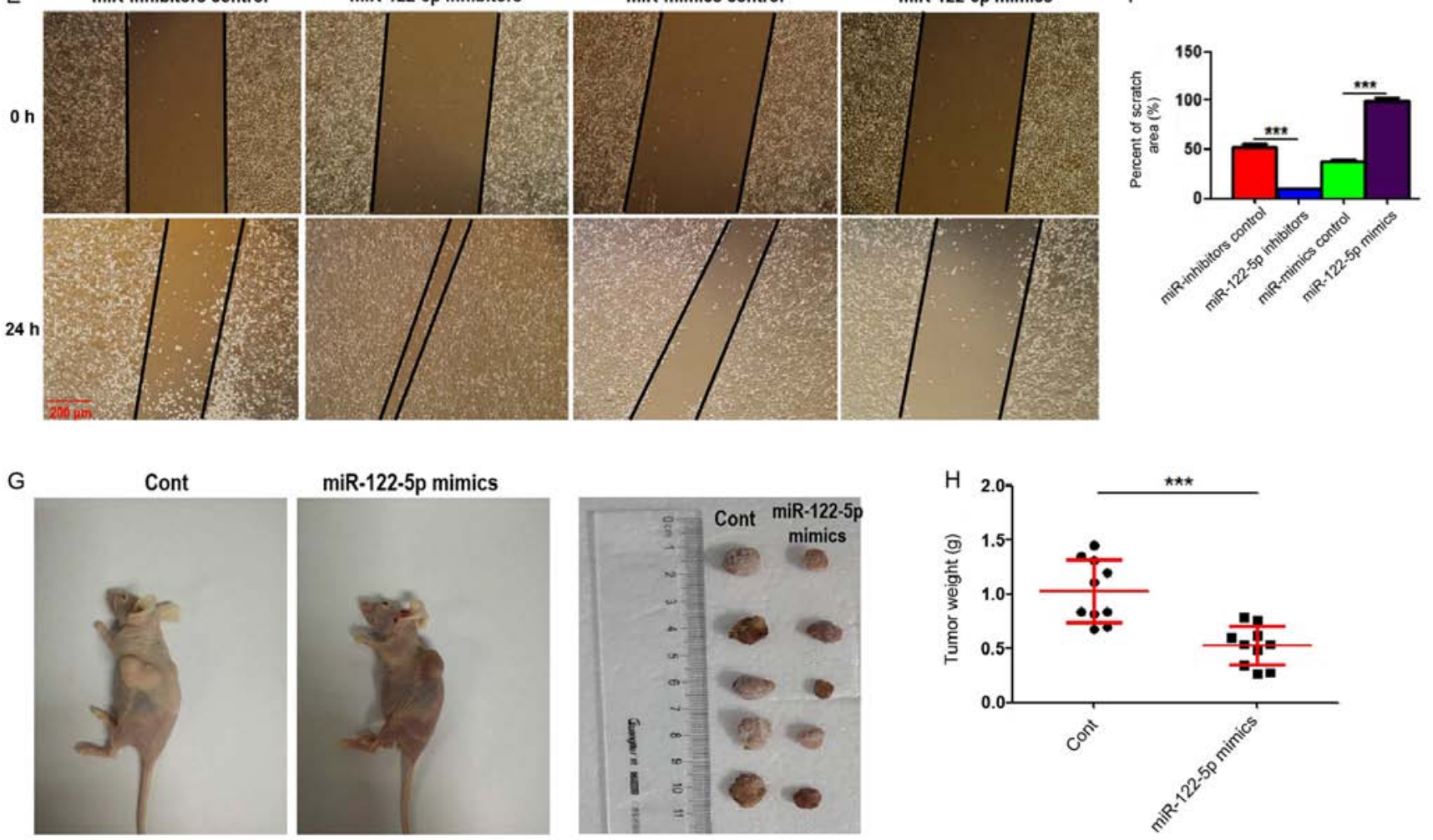

Figure 3. miR-122-5p promotes the proliferation, invasion and migration of papillary thyroid carcinoma. (A) miR-122-5p expression in K1 cells transfected with miR inhibitor control, miR-122-5p inhibitors, miR mimics control and miR-122-5p mimics. (B) Cell proliferation of treated K1 cells was evaluated using Cell Counting Kit- 8 assays. (C and D) The invasion of treated K1 cells was evaluated by Transwell assays. Scale bar, $100 \mu \mathrm{m}$. (E) The migration of treated K1 cells was assessed by scratch/wound healing assays, and images were acquired under a microscope at 0 and $24 \mathrm{~h}$. Scale bar, $200 \mu \mathrm{m}$. (F) Cell migration ratios $(24 \mathrm{~h} / 0 \mathrm{~h})$ are presented in the histogram. Results are presented as the mean \pm SEM from three independent experiments. ${ }^{* * * *} \mathrm{P}<0.001$ by one-way ANOVA. ( $\mathrm{G}$ and $\mathrm{H}$ ) miR-122-5p-overexpressing or control $\mathrm{K} 1$ cells was inoculated into nude mice. Then, 30 days later, tumour-bearing mice were sacrificed, and tumours were removed and weighed. The statistical graph indicates the quantitative results of the tumour weights ( $\mathrm{n}=10)$. Results are presented as the mean \pm SEM. ${ }^{* * *} \mathrm{P}<0.001$ by Student's t-test. miR, microRNA.

PTC tissues ( $r=-0.9028, \mathrm{P}<0.0001$; Fig. 4C). Accordingly, the regulatory effects of miR-122-5p on DUSP4 in vitro were further explored. The mRNA and protein expression levels of DUSP4 in miR-122-5p-overexpressed and silenced K1 cells were observed by RT-qPCR and western blotting. As revealed in Fig. 4D-F, both the mRNA and protein levels of DUSP4 were decreased in the miR-122-5p-overexpressed cells and increased in the miR-122-5p-silenced cells. Next, the significance of miR-122-5p in DUSP4 luciferase activity was further clarified. The luciferase reporter assay revealed that miR-122-5p overexpression reduced the luciferase activity of K1 cells, which did not occur in the pMIR-DUSP4-MUT plasmid-transfected cells that lacked the effective binding region of DUSP4 (Fig. 4G).

\section{Discussion}

The key role of miRNAs in tumorigenesis has attracted great attention. Notably, an increasing number of miRNAs have been confirmed to be implicated in PTC. Some miRNAs have negative effects in PTC, for example, miR-222 can promote the invasion and metastasis of PTC (18), and miR-146b-5p can enhance the migration and invasion of PTC cells (19). By contrast, some miRNAs have positive effects in PTC, for example, miR-599 has been found to promote apoptosis and inhibit proliferation and the epithelial-mesenchymal transition in PTC cells (3). In addition, miR-215 can suppress the proliferation, migration and invasion of PTC cells (4). Similar reports also confirmed the inhibitory effect of miR-let-7e, 
A

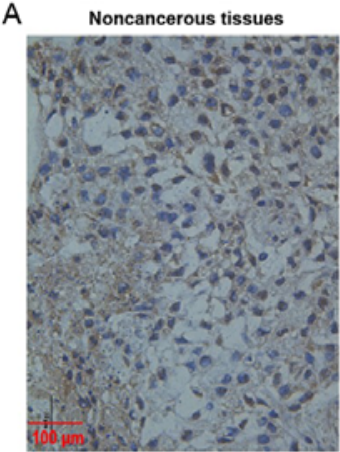

D

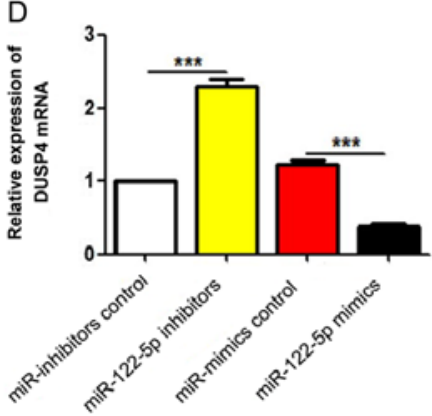

PTC tissues

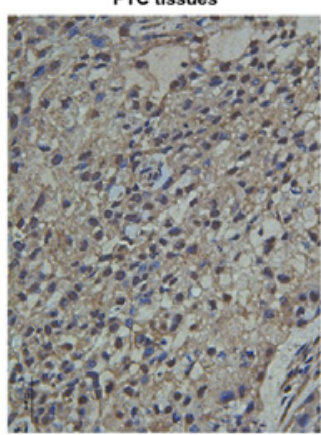

$\mathrm{E}$
B

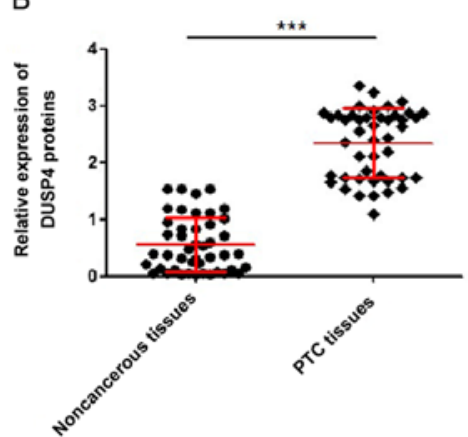

C

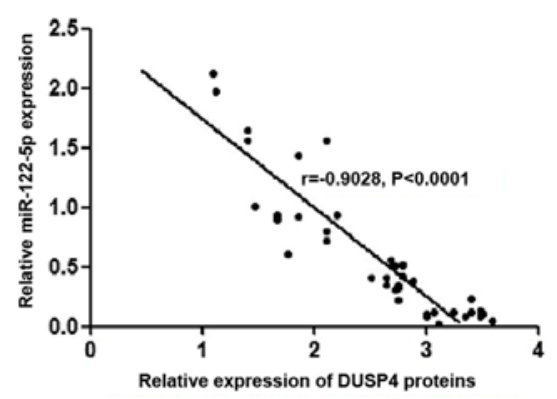

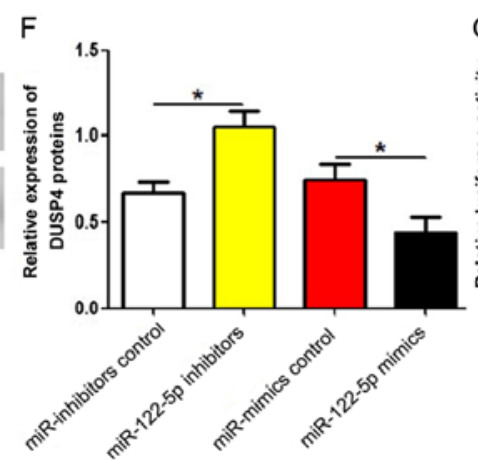

G
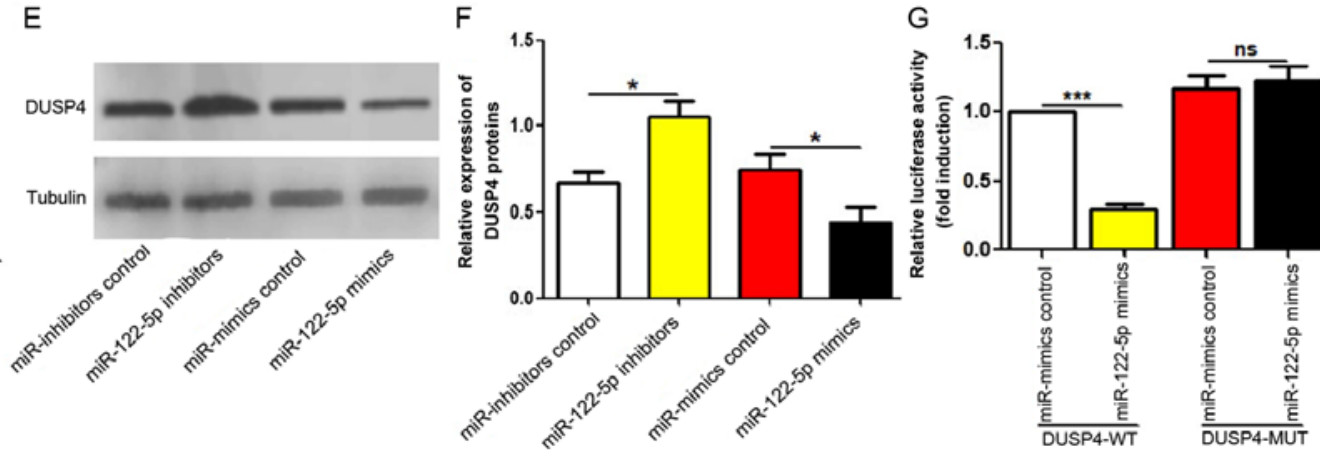

Figure 4. miR-122-5p suppresses DUSP4 expression. (A and B) DUSP4 protein expression in 45 pairs of PTC tissues and adjacent non-cancerous tissues were evaluated by immunohistochemical assay. Scale bar, $100 \mu \mathrm{m}$. The quantitative results are presented as the median with interquartile range. ${ }^{* * *} \mathrm{P}<0.001$ by Wilcoxon signed-rank test. (C) The correlation between DUSP4 protein levels and miR-122-5p mRNA levels in PTC tissues was analyzed. $\mathrm{n}=45$, $\mathrm{r}=-0.9028$, $\mathrm{P}<0.0001$ by Pearson's correlation analysis. (D-F) After transfection with corresponding plasmids for $72 \mathrm{~h}$, DUSP4 levels in treated K1 cells were evaluated by reverse transcription-quantitative PCR or western blotting. The results in F represent the protein expression of DUSP4. The relative expression refers to the ratio of DUSP4 to tubulin. ${ }^{*} \mathrm{P}<0.05$ and ${ }^{* * * *} \mathrm{P}<0.001$ by one-way ANOVA. (G) After transfection with constructed pMIR-DUSP4-WT or pMIR-DUSP4-MUT plasmid (including miR-122-5p mimics and miR mimics control) for $48 \mathrm{~h}$, the luciferase activity of each group was analyzed by dual-luciferase reporter assays. Results are presented as the mean \pm SEM from three independent experiments. ${ }^{* * *} \mathrm{P}<0.001$ by one-way ANOVA. miR, microRNA; DUSP4, dual specificity phosphatase 4; PTC, papillary thyroid carcinoma; WT, wild-type; MUT, mutant; ns, no significance.

\section{DUSP4 5'-AUAAAAGCAAACAGAACACUCCA miR-122-5p 3'-GUUUGUGGUAACAGUGUGAGGU}

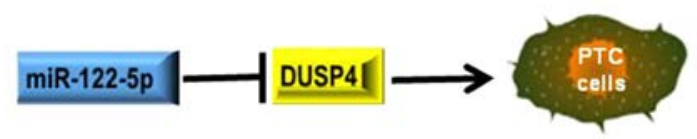

Figure 5. Schematic diagram showing the underlying mechanism of miR-122-5p in the inhibition of PTC oncogenesis via the regulation of DUSP4 expression. miR, microRNA; PTC, papillary thyroid carcinoma; DUSP4, dual specificity phosphatase 4 .

miR-200b, miR-188-5p and miR-524-5p on PTC oncogenesis (20-23). In the present study, miR-122-5p was focused on, which is widely reported to suppress the growth and function of tumours (5-7) and specifically bind to the 3'-UTR of PTC promoters (7). However, there is a lack of studies regarding the role of miR-122-5p in PTC. As anticipated, miR-122-5p was decreased in PTC tissues and cell lines compared with non-cancerous tissues and a normal thyroid epithelial cell line, respectively. Furthermore, with the growth and metastasis of PTC, miR-122-5p expression in cancer tissues was further reduced. The association analyses between miR-122-5p expression and clinicopathological characteristics revealed that the reduced expression of miR-122-5p indicated poor overall survival. Previous studies have revealed several
miRNAs that can predict the prognosis of PTC, including miR-599 and miR-215 (3,4,24,25). Thus, the aforementioned data suggested that miR-122-5p may be a novel promising prognostic biomarker for PTC.

Functional assays revealed that overexpression of miR-122-5p inhibited the proliferation, invasion and migration of PTC in vitro. Conversely, knockdown of miR-122-5p promoted the proliferation, invasion and migration of PTC in vitro. Nude mouse xenograft experiments revealed that miR-122-5p overexpression suppressed PTC growth in vivo. Several miRNAs have been revealed to inhibit the carcinogenesis of PTC, such as miR-let-7e and miR-200b (20-23). These data indicated that miR-122-5p may have a potential therapeutic effect on PTC.

A previous bioinformatics study revealed that the 3'-UTR of DUSP4 mRNA has a specific binding sequence for miR-122-5p (7), deducing that DUSP4 may be a downstream target of miR-122-5p. The expression of miR-122-5p was negatively associated with DUSP4 expression in PTC tissues, which confirmed the negative regulation of DUSP4 expression by miR-122-5p. Furthermore, cytology experiments revealed that miR-122-5p overexpression inhibited DUSP4 expression, while miR-122-5p knockdown promoted DUSP4 expression. Notably, a luciferase reporter assay demonstrated that miR-122-5p can negatively regulate the luciferase activity of the DUSP4 reported in PTC cells by binding to the 3'-UTR, which explains the inhibitory effect of miR-122-5p on DUSP4 
expression in PTC cells. DUSP4 has been demonstrated to contribute to the occurrence and development of PTC (12). The aforementioned results indicated that the negative regulation of DUSP4 at least partially accounts for the roles of miR-122-5p in PTC. The working model of the study is presented in Fig. 5. However, the intrinsic mechanism underlying DUSP4-regulated PTC carcinogenesis requires further investigation.

In conclusion, the present study confirmed that miR-122-5p is a tumour-suppressor miRNA in PTC and a promising prognostic biomarker of PTC. Moreover, miR-122-5p mimics may be a potential treatment strategy for PTC. Based on these results, further improvement in the diagnosis and treatment of PTC may be achieved in the future.

\section{Acknowledgements}

Not applicable.

\section{Funding}

The present study was supported by The Natural Science Foundation of Hebei Province (grant no. H2018206180).

\section{Availability of data and materials}

The data generated or analysed during this study are included in this published article.

\section{Authors' contributions}

LZ and NH conceived and designed the experiments. $\mathrm{NH}$ and YT performed the experiments, analyzed the data, and prepared the figures. YS helped with the analysis of the data. $\mathrm{LZ}$ and $\mathrm{NH}$ wrote the manuscript. $\mathrm{LZ}$ and $\mathrm{NH}$ confirm the authenticity of all the raw data. All the authors read and approved the final manuscript.

\section{Ethics approval and consent to participate}

The present study protocol was approved by the Research Ethics Committee of the Second Hospital of Hebei Medical University (Shujiazhuang, China) and written informed consent was obtained from all the participants for their tissues to be used for the purposes of this research. The clinical studies were conducted according to the principles expressed in the Declaration of Helsinki. Athymic nude mice assays were conducted in accordance with the Institutional principles for the concern and use of animals, and the corresponding protocols (including euthanasia protocol) was approved by the Institutional Animal Care and Use Committee of the Second Hospital of Hebei Medical University.

\section{Patient consent for publication}

Not applicable.

\section{Competing interests}

The authors declare that they have no competing interests.

\section{References}

1. Yang Z, Li G, Ding C, Sun W and Zhang J: Long non-coding RNA HULC exerts oncogenic activity on papillary thyroid cancer in vitro and in vivo. Artif Cells Nanomed Biotechnol 48: 326-335, 2020.

2. Wiltshire JJ, Drake TM, Uttley L and Balasubramanian SP: Systematic review of trends in the incidence rates of thyroid cancer. Thyroid 26: 1541-1552, 2016.

3. Wang DP, Tang XZ, Liang QK, Zeng XJ, Yang JB and Xu J: microRNA-599 promotes apoptosis and represses proliferation and epithelial-mesenchymal transition of papillary thyroid carcinoma cells via downregulation of Hey2-depentent Notch signaling pathway. J Cell Physiol 235: 2492-2505, 2020.

4. Han J, Zhang M, Nie C, Jia J, Wang F, Yu J, Bi W, Liu B, Sheng R, $\mathrm{He} \mathrm{G}$, et al: miR-215 suppresses papillary thyroid cancer proliferation, migration, and invasion through the AKT/GSK-3 $\beta /$ Snail signaling by targeting ARFGEF1. Cell Death Dis 10: 195, 2019.

5. Ma J, Li T, Han X and Yuan H: Knockdown of LncRNA ANRIL suppresses cell proliferation, metastasis, and invasion via regulating miR-122-5p expression in hepatocellular carcinoma. J Cancer Res Clin Oncol 144: 205-214, 2018.

6. Xu Z, Liu G, Zhang M, Zhang Z, Jia Y, Peng L, Zhu Y, Hu J, Huang R and Sun X: miR-122-5p inhibits the proliferation, invasion and growth of bile duct carcinoma cells by targeting ALDOA. Cell Physiol Biochem 48: 2596-2606, 2018.

7. Xu X, Gao F, Wang J, Tao L, Ye J, Ding L, Ji W and Chen X: MiR-122-5p inhibits cell migration and invasion in gastric cancer by down-regulating DUSP4. Cancer Biol Ther 19: 427-435, 2018.

8. Balko JM, Schwarz LJ, Bhola NE, Kurupi R, Owens P, Miller TW, Gómez H, Cook RS and Arteaga CL: Activation of MAPK pathways due to DUSP4 loss promotes cancer stem cell-like phenotypes in basal-like breast cancer. Cancer Res 73: 6346-6358, 2013.

9. Hijiya N, Tsukamoto Y, Nakada C, Tung Nguyen L, Kai T, Matsuura K, Shibata K, Inomata M, Uchida T, Tokunaga A, et al: Genomic loss of DUSP4 contributes to the progression of intraepithelial neoplasm of pancreas to invasive carcinoma. Cancer Res 76: 2612-2625, 2016.

10. Chen M, Zhang J, Berger AH, Diolombi MS, Ng C, Fung J, Bronson RT, Castillo-Martin M, Thin TH, Cordon-Cardo C, et al: Compound haploinsufficiency of Dok2 and Dusp4 promotes lung tumorigenesis. J Clin Invest 129: 215-222, 2019.

11. Gröschl B, Bettstetter M, Giedl C, Woenckhaus M, Edmonston T, Hofstädter F and Dietmaier W: Expression of the MAP kinase phosphatase DUSP4 is associated with microsatellite instability in colorectal cancer (CRC) and causes increased cell proliferation. Int J Cancer 132: 1537-1546, 2013.

12. Ma B, Shi R, Yang S, Zhou L, Qu N, Liao T, Wang Y, Wang Y and Ji Q: DUSP4/MKP2 overexpression is associated with BRAF(V600E) mutation and aggressive behavior of papillary thyroid cancer. Onco Targets Ther 9: 2255-2263, 2016.

13. Heinemann FG, Tolkach Y, Deng M, Schmidt D, Perner S, Kristiansen G, Müller SC and Ellinger J: Serum miR-122-5p and miR-206 expression: Non-invasive prognostic biomarkers for renal cell carcinoma. Clin Epigenetics 10: 11, 2018.

14. Cheng G, Li Y, Liu Z and Song X: The microRNA-429/DUSP4 axis regulates the sensitivity of colorectal cancer cells to nintedanib. Mol Med Rep 23: 1, 2012.

15. Livak KJ and Schmittgen TD: Analysis of relative gene expression data using real-time quantitative PCR and the 2(-Delta Delta C(T)) method. Methods 25: 402-408, 2001.

16. Masson D, Rioux-Leclercq N, Fergelot P, Jouan F, Mottier S, Théoleyre S, Bach-Ngohou K, Patard JJ and Denis MG: Loss of expression of TIMP3 in clear cell renal cell carcinoma. Eur J Cancer 46, 1430-1437, 2010.

17. Kong QF, Lv B, Wang B, Zhang XP, Sun HJ and Liu J: Association of von Willebrand factor ( $\mathrm{vWF}$ ) expression with lymph node metastasis and hemodynamics in papillary thyroid carcinoma. Eur Rev Med Pharmacol Sci 24: 2564-2571, 2020.

18. Huang Y, Yu S, Cao S, Yin Y, Hong S, Guan H, Li Y and Xiao H: MicroRNA-222 promotes invasion and metastasis of papillary thyroid cancer through targeting protein phosphatase 2 regulatory subunit B alpha expression. Thyroid 28: 1162-1173, 2018.

19. Lima CR, Geraldo MV, Fuziwara CS, Kimura ET and Santos MF: MiRNA-146b-5p upregulates migration and invasion of different papillary thyroid carcinoma cells. BMC Cancer 16: 108, 2016. 
20. Ding C, Yu H, Shi C, Shi T, Qin H and Cui Y: MiR-let-7e inhibits invasion and magration and regulates HMGB1 expression in papillary thyroid carcinoma. Biomed Pharmacother 110 528-536, 2019.

21. Zhou B, Xu J, Chen Y, Gao S, Feng X and Lu X: miR-200b/ c-RAP1B axis represses tumorigenesis and malignant progression of papillary thyroid carcinoma through inhibiting the NF-кB/Twistl pathway. Exp Cell Res 387: 111785, 2020.

22. Zhou P, Irving A, Wu H, Luo J, Aguirre J, Costa M, Khamsuree M, Gerads N and Liu W: Validation of MicroRNA-188-5p inhibition power on tumor cell proliferation in papillary thyroid carcinoma. Cell Transplant 29: 963689720918300, 2020.

23. Liu H, Chen $X$, Lin T, Chen $X$, Yan J and Jiang $S$ : MicroRNA-524-5p suppresses the progression of papillary thyroid carcinoma cells via targeting on FOXE1 and ITGA3 in cell autophagy and cycling pathways. J Cell Physiol 234: 18382-18391, 2019.
24. Santiago K, Chen Wongworawat Y and Khan S: Differential MicroRNA-signatures in thyroid cancer subtypes. J Oncol 2020: 2052396, 2020

25. Jiang K, Li G, Chen W, Song L, Wei T, Li Z, Gong R, Lei J, Shi $\mathrm{H}$ and Zhu J: Plasma exosomal miR-146b-5p and miR-222-3p are potential biomarkers for lymph node metastasis in papillary thyroid carcinomas. Onco Targets Ther 13: 1311-1319, 2020.

This work is licensed under a Creative Commons Attribution-NonCommercial-NoDerivatives 4.0 International (CC BY-NC-ND 4.0) License. 